\title{
Eicheons instead of Black holes
}

\author{
S L Cherkas ${ }^{1,3}$ (i) and V L Kalashnikov ${ }^{2}$ \\ ${ }^{1}$ Institute for Nuclear Problems, Bobruiskaya 11, Minsk 220030, Belarus \\ ${ }^{2}$ Facoltá di Ingegneria dell'Informazione, Informatica e Statistica, Sapienza Universitá di Roma, Via \\ Eudossiana 18 00189—Roma, RM, Italy \\ E-mail: cherkas@inp.bsu.by
}

Received 8 April 2020, revised 24 June 2020

Accepted for publication 7 July 2020

Published 29 July 2020

\begin{abstract}
A new spherically-symmetric solution for a gravitational field is found in the conformallyunimodular metric. It is shown, that the surface of the black hole horizon in the standard Schwarzschild metric can be squeezed to a point by converting coordinates to the conformally-unimodular metric. In this new metric, there is no black hole horizon, while the naked singularity corresponds to a point massive particle. The reason for the study of this particular gauge (i.e., conformally-unimodular metric) is its relation to the vacuum energy problem. That aims to relate it to other physical phenomena (including black holes), and one could argue that they should be considered in this particular metric. That means the violation of the gauge invariance of the general theory of relativity. As a result, the nonsingular 'eicheons" ${ }^{4}$ appear as the non-point compact objects with different masses and structures. They are a final product of the stellar collapse, with the masses exceeding the Tolman-OppenheimerVolkoff limit.
\end{abstract}

Keywords: black holes, supermassive black holes, coordinate singularity, conformallyunimodular metric, gauge invariance violation, vacuum energy

\section{Introduction}

One of the most intriguing objects in the theory of general relativity (GR) is the 'black hole' (BH) [2, 3], which is a result of the collapse of astrophysical objects with the masses exceeding the Tolman-Volkov-Oppenheimer (TVO) limit $[4,5]$. The gravitational waves registered recently are considered as a result of the collision of massive BHs [6]. Direct astrophysical observations also indicate the extremely compact supermassive objects in the galactic cores [7] identified with $\mathrm{BH}$. However, such $\mathrm{BH}$ evidences should be considered with caution because they suggest only a presence of some compact massive astrophysical object possessing the $\mathrm{BH}$

\footnotetext{
3 Author to whom any correspondence should be addressed.

4 The term 'eicheon' refers to the fundamental work 'Gravitation und Elektrizität' by Hermann Weyl where the concept of 'gauge field theory' ('Eichfeldtheorie') was invented for the first time (e.g., see [1]). We would emphasize by this term the decisive role of the gauge conditions in our theory predicting an existence of extremely compact but nonsingular astrophysical objects. Moreover, the connotation with 'Eichel' (that means an acorn in German) implies that 'eicheon' can have an internal structure and a solid-like 'surface'.
}

properties for an external observer, but with the wholly unknown internal structure.

The strange properties of $\mathrm{BH}$ forced many researchers (including A. Einstein [8]) to question the $\mathrm{BH}$ reality and consider these objects as a pathological artifact of GR. Several discouraging facts are well-known:

(1) The first issue is the presence of $\mathrm{BH}$ singularity with an infinitely large density, which is physically questionable. In order to avoid a singular state, the different modifications of GR have been offered by taking into account torsion (see, for example, [9]); space-time curvature limitations [10]; or considering the gravitation as a physical tensor field which Requires gauge invariance violation and non-zero graviton mass [11]; and, at least, development of quantum theories gravity, e.g., loop quantum gravity [12]. On the other hand, the $\mathrm{BH}$ singularity could be justified because it is 'dressed,' i.e., surrounded by a horizon, making it invisible for an outside observer (the so-called 'cosmic censorship' principle [13]). The BH singularity found a treatment within the framework of GR (the concept of a so-called 'regular BH' [14, 15]) through the modification of the energy conditions on the stress-energy tensor of matter $[16,17]$. In particular, the impact of nonlinear electrodynamics 
and non-Abelian gauge fields on the $\mathrm{BH}$ formation and structure were studied [18-20], and the limits of the BH 'non-hair' theorem were discussed [21].

(2) The physical status of the 'event horizon' itself could also raise the questions. However, from GR, it is merely a 'one-sided membrane' ('no-return horizon') for the free-falling observer. Nevertheless, a fact of the event horizon existence is doubted both from classical and quantum viewpoints. For example, the horizon formation relates to the stability of ultra-compact states of a substance [5]. The existence of such exotic stable phases (e.g., free-quark phase [22]) could explain the phenomenon of ultra-compact objects but with the size larger than the horizon. Then, the concept of the event horizon, as well as the unlimited gravitational collapse, are declared physically meaningless in the field formulation of gravity with a massive graviton [11, 23, 24]. However, the existence of ultra-compact objects, which are finely larger than $\mathrm{BH}$, is not disclaimed $[25,26]$. A quantum view on the horizon issue reveals an 'information paradox,' and a 'noncloning' of quantum states, as well as the thermodynamical problems [27-30].

Assuming the modernization of GR in its relation with the 'no-BH'-hypothesis refers to the synthesis of gravity with quantum mechanics. That raises the question: what is the direction of such modernization? In this regard, one can recall the known statement by D.I. Blochintsev: 'Number of facts is always enough, but fantasy is insufficient.' [31].

The key fact indicating a possible path in the forest of the alternative gravity theories is the vacuum energy problem. In GR, any spatially uniform energy density (including that of zero-point fluctuations of the quantum fields) causes the expansion of the Universe. Using the Planck level of UVcutoff results in the Planckian vacuum energy density $\rho_{v a c} \sim M_{p}^{4}$ [32], which leads to the Universe expanding with the Planckian rate [33]. In this sense, the vacuum energy problem is an observational fact [34].

One of the possible solutions is to build a theory of gravity, allowing an arbitrarily reference level of energy density. One such theory has long been known. That is the unimodular gravity [35-39], which admits an arbitrary cosmological constant. However, under using of the comoving momentums cutoff, the vacuum energy density scales with time as radiation [34], but not as the cosmological constant.

Recently, another theory has been suggested [40], which considers the Friedmann equation defined up to some arbitrary constant. This constant corresponds to the invisible radiation and, thus, can compensate the vacuum energy. In this case, one could ask why the $\boldsymbol{k}$-cutoff of comoving momentums is used instead of, for instance, a cutoff of physical momentums related to $\boldsymbol{p}=\boldsymbol{k} / \boldsymbol{a}$ ( $a$ is the Universe scale factor)? The answer could be that it is relatively simple to construct a theory with the $\boldsymbol{k}$-cutoff, but it is challenging to introduce the $\boldsymbol{p}$-cutoff fundamentally. For instance, merely considering gravity on a lattice gives rather fundamental theory with comoving momentums restricted by the period of a lattice.

The next noteworthy fact of GR is the absence of a vacuum state, which is invariant relative to the general transformation of coordinates. It indicates the violation of gauge invariance at a quantum level ${ }^{5}$, but one could assume that the gauge invariance should be broken at the classical level in GR, as well. In particular, the five-vector theory of gravity (FVT) [40] assumes the gauge invariance violation in GR by constraining the class of all possible metrics in varying the standard Einstein-Hilbert action. A question arises, how the classical Schwarzschild solution looks in this class of metrics? The purpose of this work is to elucidate the nature of compact astrophysical objects in this limited class of conformally-unimodular metrics.

\section{Violation of gauge invariance in a framework of FVT}

The observational fact, that the bulk of vacuum energy density does not affect the expansion of the Universe, points out a gravity theory, in which the reference level of energy density could be chosen arbitrarily. Such a theory arises if one varies the standard Einstein-Hilbert action over not all possible space-time metrics $g_{\mu \nu}$, but over some class of conformallyunimodular metrics ${ }^{6}$ [40]

$$
\begin{aligned}
d s^{2} \equiv & g_{\mu \nu} d x^{\mu} d x^{\nu}=a^{2}\left(1-\partial_{m} P^{m}\right)^{2} d \eta^{2} \\
& -\gamma_{i j}\left(d x^{i}+N^{i} d \eta\right)\left(d x^{j}+N^{j} d \eta\right)
\end{aligned}
$$

where $x^{\mu}=\{\eta, \boldsymbol{x}\}, \eta$ is conformal time, $\gamma_{i j}$ is a spatial metric, $a=\gamma^{1 / 6}$ is a locally defined scale factor, and $\gamma=\operatorname{det} \gamma_{i j}$. The spatial part of the interval (1) reads as

$$
d l^{2} \equiv \gamma_{i j} d x^{i} d x^{j}=a^{2}(\eta, \boldsymbol{x}) \tilde{\gamma}_{i j} d x^{i} d x^{j},
$$

where $\tilde{\gamma}_{i j}=\gamma_{i j} / a^{2}$ is a matrix with the unit determinant.

The interval (1) is similar formally to the ADM one [41], but with the lapse function $N$ changed by the expression $1-\partial_{m} P^{m}$, where $P^{m}$ is a three-dimensional (relatively rotations) vector, and $\partial_{m}$ is a conventional particular derivative.

The starting point is the standard Einstein-Hilbert action [42]

$$
S_{\text {grav }}=-\frac{M_{p}^{2}}{12} \int \mathcal{G} \sqrt{-g} d^{4} x
$$

where $\mathcal{G}=g^{\alpha \beta}\left(\Gamma_{\alpha \nu}^{\rho} \Gamma_{\beta \rho}^{\nu}-\Gamma_{\alpha \beta}^{\nu} \Gamma_{\nu \rho}^{\rho}\right), \quad$ and $\quad M_{p}=\sqrt{\frac{3}{4 \pi G}}=$ $1.065 \times 10^{-8} \mathrm{~kg}$ is the reduced Planck mass. The variation of (3) over vectors $\boldsymbol{P}, \boldsymbol{N}$ and 3-metric ${ }^{7} \gamma_{i j}$ leads to the FVT

\footnotetext{
5 As was found, most of the symmetries in nature are violated. The exception is the color symmetry of the quantum chromodynamics.

6 In this gauge, a space-time metric is presented as a product of a common multiplier by a 4-dimensional matrix with a determinant equal to -1 , including a 3-dimensional spatial block with unit determinant.

7 Three dimensional spatial metric tensor can be written as the three-vectors triad. Thus 5-vectors appear in theory.
} 
equations:

$$
\begin{aligned}
& \frac{\partial g^{\mu \nu}}{\partial \gamma_{i j}}\left(\frac{(\partial \mathcal{G} \sqrt{-g})}{\partial g^{\mu \nu}}-\partial_{\lambda} \frac{\partial(\mathcal{G} \sqrt{-g})}{\partial\left(\partial_{\lambda} g^{\mu \nu}\right)}-\frac{6}{M_{p}^{2}} T_{\mu \nu} \sqrt{-g}\right)=0, \\
& \frac{\partial g^{\mu \nu}}{\partial N^{i}}\left(\frac{\partial(\mathcal{G} \sqrt{-g})}{\partial g^{\mu \nu}}-\partial_{\lambda} \frac{\partial(\mathcal{G} \sqrt{-g})}{\partial\left(\partial_{\lambda} g^{\mu \nu}\right)}-\frac{6}{M_{p}^{2}} T_{\mu \nu} \sqrt{-g}\right)=0, \\
& \frac{\partial g^{\mu \nu}}{\partial\left(\partial_{j} P^{i}\right)} \frac{\partial}{\partial x^{j}}\left(\frac{\partial(\mathcal{G} \sqrt{-g})}{\partial g^{\mu \nu}}-\partial_{\lambda} \frac{\partial(\mathcal{G} \sqrt{-g})}{\partial\left(\partial_{\lambda} g^{\mu \nu}\right)}\right. \\
& \left.-\frac{6}{M_{p}^{2}} T_{\mu \nu} \sqrt{-g}\right)=0,
\end{aligned}
$$

where the energy momentum tensor $T_{\mu \nu}=\frac{\delta S_{m}}{\delta_{g^{\mu \nu}}}$ is introduced. The last equation (4) is weaker than the corresponding Hamiltonian constraint of GR. On the other hand, the restrictions $\boldsymbol{\nabla}(\boldsymbol{\nabla} \cdot \boldsymbol{P})=0, \boldsymbol{\nabla}(\boldsymbol{\nabla} \cdot \boldsymbol{N})=0$ on the Lagrange multipliers arise in FVT. Using the gauge $\boldsymbol{\nabla} \cdot \boldsymbol{N}=0$ provides the Hamiltonian constraint fulfillment up to some constant [40].

\section{A spherically symmetric static gravitational field}

The spherically symmetric metrics belonging to the class (1) reads as:

$$
\begin{aligned}
d s^{2} & =a^{2}\left(d \eta^{2}-\tilde{\gamma}_{i j} d x^{i} d x^{j}\right) \\
& =e^{2 \alpha}\left(d \eta^{2}-e^{-2 \lambda}(d \boldsymbol{x})^{2}-\left(e^{4 \lambda}-e^{-2 \lambda}\right)(\boldsymbol{x} d \boldsymbol{x})^{2} / r^{2}\right),
\end{aligned}
$$

where $r=|x|$ and $a=\exp \alpha, \lambda$ are the functions of $\eta, r$. The matrix $\tilde{\gamma}_{i j}$ with the unit determinant is expressed through $\lambda(\eta, r)$. Thus, for the spherically symmetric case, the equations (4) take the form

$$
\begin{aligned}
\mathcal{H}= & e^{2 \alpha}\left(-\frac{1}{2} \alpha^{\prime 2}+\frac{1}{2} \lambda^{\prime 2}-\frac{e^{2 \lambda}}{6 r^{2}}+\frac{e^{2 \alpha}}{M_{p}^{2}} \rho\right. \\
& +e^{-4 \lambda}\left(\frac{1}{6 r^{2}}-\frac{4}{3} \partial_{r} \alpha \partial_{r} \lambda+\frac{1}{6} \partial_{r} \alpha^{2}\right. \\
& +\frac{2 \partial_{r} \alpha}{3 r}+\frac{1}{3} \partial_{r, r} \alpha+\frac{7}{6} \partial_{r} \lambda^{2} \\
& \left.\left.-\frac{5 \partial_{r} \lambda}{3 r}-\frac{1}{3} \partial_{r, r} \lambda\right)\right)=\text { const },
\end{aligned}
$$

$$
\begin{aligned}
\mathcal{P}= & e^{2 \alpha}\left(-\partial_{r} \alpha\left(\alpha^{\prime}+2 \lambda^{\prime}\right)-\partial_{r} \lambda^{\prime}+\partial_{r} \alpha^{\prime}\right. \\
& \left.-\left(3 / r-3 \partial_{r} \lambda\right) \lambda^{\prime}\right)=0,
\end{aligned}
$$

$$
\begin{aligned}
& \alpha^{\prime \prime}+\alpha^{\prime 2}+\lambda^{\prime 2}=e^{-4 \lambda}\left(-4 \partial_{r} \alpha \partial_{r} \lambda+\partial_{r} \alpha^{2}\right. \\
& +\frac{2 \partial_{r} \alpha}{r}+\partial_{r, r} \alpha+\frac{7}{3} \partial_{r} \lambda^{2} \\
& \left.-\frac{10 \partial_{r} \lambda}{3 r}-\frac{2}{3} \partial_{r, r} \lambda+\frac{1}{3 r^{2}}\left(1-e^{6 \lambda}\right)\right)+\frac{e^{2 \alpha}}{M_{p}^{2}}(3 p-\rho)
\end{aligned}
$$

$$
\begin{aligned}
\lambda^{\prime \prime}+2 \alpha^{\prime} \lambda^{\prime}= & \frac{2}{3} e^{-4 \lambda}\left(-\partial_{r} \alpha \partial_{r} \lambda-\partial_{r} \alpha^{2}+\partial_{r, r} \alpha\right. \\
& +\partial_{r} \lambda^{2}-\frac{1}{2} \partial_{r, r} \lambda-\frac{1}{r} \partial_{r} \alpha \\
& \left.-\frac{1}{r} \partial_{r} \lambda+\frac{1}{2 r^{2}}\left(e^{6 \lambda}-1\right)\right),
\end{aligned}
$$

where prime denotes differentiation over $\eta$. Equation (6) is the Hamiltonian constraint, but it includes an arbitrary constant now. If this constant equals zero, one returns to GR. Equation (7) follows from the momentum constraint. The expressions (8), (9) are the equations of motion.

Differentiation of the constraints over time $\eta$ results in the following equations

$$
\begin{gathered}
\mathcal{H}^{\prime}=\frac{1}{3 r^{2}} \partial_{r}\left(e^{-4 \lambda} r^{2} \mathcal{P}\right), \\
\mathcal{P}^{\prime}=\partial_{r} \mathcal{H},
\end{gathered}
$$

which are satisfied if the equations of motion (8), (9) are fulfilled, and, besides, the following equations for the energy density and pressure are enforced:

$$
\rho^{\prime}+3(p+\rho) \alpha^{\prime}=0, \quad \partial_{r} p+(p+\rho) \partial_{r} \alpha=0 .
$$

In GR, the equations (12) arise from the Bianchi identities resulting in $D^{\mu} T_{\mu \nu}=0$. In the FVT case, the relations (12) arise from the requirement of the constraints conservation in the time (10), (11). Generally, the equations (10), (11) satisfy not only $\mathcal{H}=0, \mathcal{P}=0$, as in GR, but weaker conditions $\mathcal{H}=$ const, $\mathcal{P}=0$, as reflected in equation (6). A constant on the right hand side of equation (6) compensates the bulk of the vacuum energy, and, after the compensation (if it is exact), the equations become the same as in GR. All this take a place in the conformo-unimodular metric (5), in which we will find the Schwarzschild solution, assuming the time derivatives, as well as pressure and density equal to zero in equations (6-9). Expressing the derivatives $\partial_{r, r} \lambda, \partial_{r, r} \alpha$ from equations (8), (9) and substituting them into (6) under the const $=0$, one finds

$$
\begin{gathered}
-3 r^{2}\left(\frac{d \alpha}{d r}\right)^{2}+4 r \frac{d \alpha}{d r}\left(r \frac{d \lambda}{d r}-1\right) \\
-\left(r \frac{d \lambda}{d r}-1\right)^{2}+e^{6 \lambda}=0
\end{gathered}
$$

To obtain a solution of the equations (8), (9), (13), let us make the following substitution

$$
\lambda=\alpha+\ln \left(\left(1-e^{2 \alpha}\right) r / r_{g}\right),
$$

where the Schwarzschild radius is introduced for the sake of dimensionless of the expressions under logarithm.

As a result, equation (13) takes the form:

$$
r^{4} e^{4 \alpha}\left(e^{2 \alpha}-1\right)^{8}-4\left(\frac{d \alpha}{d r}\right)^{2} r_{g}^{6}=0
$$

and has the solution

$$
\alpha(r)=\ln \left(f^{(-1)}\left(\frac{r^{3}-r_{0}^{3}}{6 r_{g}^{3}}\right)\right),
$$




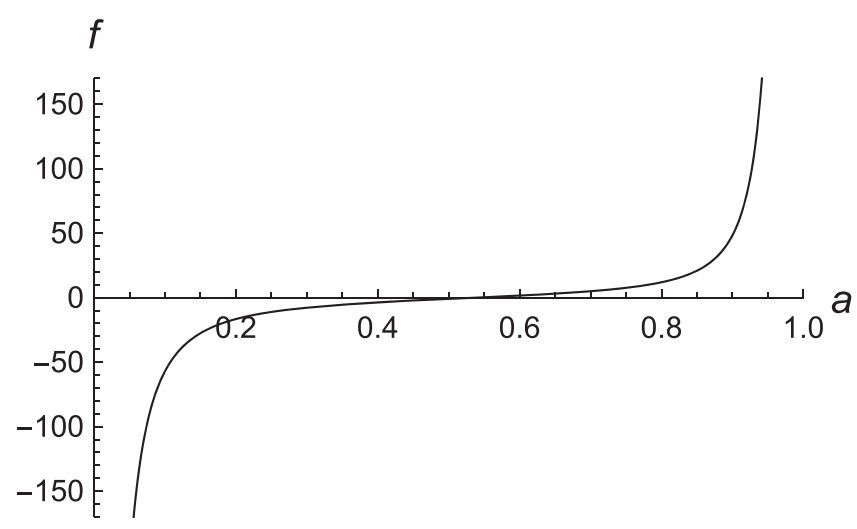

Figure 1. Plot of the function $f(a)$ defined by equation (17).

where $f^{-1}$ is the inverse function of

$f(a)=2 \ln \left(\frac{a^{2}}{1-a^{2}}\right)+\frac{30 a^{4}-12 a^{6}-22 a^{2}+3}{6 a^{2}\left(a^{2}-1\right)^{3}}$

and $r_{0}$ is an integration constant. The function $f(a)$, which maps an interval $(0,1)$ into $\mathbb{R}$, is mutually single-valued function shown in figure 1. Using (16), (17) and the rules of the differentiation of the inverse function allows calculating

$$
\begin{aligned}
\frac{d \alpha}{d r} & =\frac{r^{2}}{2 r_{g}^{3} f^{(-1)}(y) f^{\prime}\left(f^{(-1)}(y)\right)} \\
& =\frac{r^{2} f^{(-1)}(y)^{2}\left(f^{(-1)}(y)^{2}-1\right)^{4}}{2 r_{g}^{3}}=\frac{8 e^{6 \alpha} r^{2} \sinh ^{4} \alpha}{r_{g}^{3}},
\end{aligned}
$$

where $y=\frac{r^{3}-r_{0}^{3}}{6 r_{g}^{3}}$. Similar calculations give

$$
\begin{aligned}
& \frac{d \lambda}{d r}=\frac{8 e^{6 \alpha} r^{2} \sinh ^{4} \alpha(\operatorname{coth} \alpha+2)}{r_{g}^{3}}+\frac{1}{r}, \\
\frac{d^{2} \alpha}{d r^{2}}= & \frac{16}{r_{g}^{6}} e^{6 \alpha} r \sinh ^{4} \alpha \\
& \times\left(8 e^{6 \alpha} r^{3} \sinh ^{3} \alpha(3 \sinh \alpha+2 \cosh \alpha)+r_{g}^{3}\right), \\
\frac{d^{2} \lambda}{d r^{2}}= & \frac{64 e^{12 \alpha} r^{4} \sinh ^{6} \alpha(7 \sinh (2 \alpha)+8 \cosh (2 \alpha)-5)}{r_{g}^{6}} \\
& -\frac{1}{r^{2}}+\frac{16 e^{6 \alpha} r \sinh ^{4} \alpha(\operatorname{coth} \alpha+2)}{r_{g}^{3}} .
\end{aligned}
$$

Substitution of equations (18), (19), (20), (21) into equations (6), (7), (8), (9) demonstrates that the lasts are satisfied at $p=\rho=0$, and const $=0$. Thus, equations (14), (16), (17) are the exact spherically-symmetric static solution of the Einstein equations in vacuum. From the physical viewpoint, it appears that const in equation (5) compensates a vacuum energy of quantum fields.

The function $\alpha$ is not singular everywhere, as it is shown in figure 2(a), whereas the function $\lambda$, describing the deviation of conformally-unimodular metric geometry from the Schwarzschild one, is singular only at $r=0$.
Let us compare the solution (14), (16) with the canonical Schwarzschild one which is [42]

$$
\begin{aligned}
d s^{2}= & \left(1-r_{g} / R\right) d t^{2}-\left(1-r_{g} / R\right)^{-1} d R^{2} \\
& -R^{2}\left(d \theta^{2}+\sin ^{2} \theta d \phi^{2}\right) .
\end{aligned}
$$

For this aim, we rewrite the interval (5) in the spherical coordinates

$$
d s^{2}=e^{2 \alpha}\left(d \eta^{2}-d r^{2} e^{4 \lambda}-e^{-2 \lambda} r^{2}\left(d \theta^{2}+\sin ^{2} \theta d \phi^{2}\right)\right) .
$$

The solutions (22) and (23) of the Einstein equations should be interrelated by the transformation of coordinates $t=\eta, \quad R=R(r)$, which gives another way to deduce equations (14), (15). Actually, equating the coefficients at $d t^{2}=d \eta^{2}, d \theta^{2}$ and $\sin ^{2} \theta d \phi^{2}$, as well as the radial terms in the intervals (22), (23) gives the equations

$$
\begin{gathered}
1-r_{g} / R=e^{2 \alpha}, \\
R^{2}=r^{2} e^{-2 \lambda+2 \alpha}, \\
\left(1-r_{g} / R\right)^{-1}\left(\frac{d R}{d r}\right)^{2}=e^{4 \lambda+2 \alpha} .
\end{gathered}
$$

The relations (24), (25) result in the expressions (14) and $R(r)=r_{g}\left(1-e^{2 \alpha}\right)^{-1}$, which give (15) after substitution in (26). As is shown in figure 2(b), the solutions (14), (16) describe only a part of the external space $r_{g}^{+}$of the Schwarzschild solution by virtue of $\lim _{r \rightarrow 0} R(r) \gtrsim r_{g}$. Figure 3 illustrates this fact in the following way. Let there is a space filled with the Schwarzschild BHs. Then, by inverse coordinates transformation having the form $r(R)$ in the vicinity of each hole, one can squeeze holes into the nodes $r=0$ and consider that point particle is placed in each node. The space-time obtained in such a way will represent a single causally connected region. That corresponds to the existence of impenetrable surface at $R(0)>r_{g}$ in the metric (22). This situation resembles that in theory with massive graviton, where the physical singularity corresponds to $R=r_{g}[24,26]$.

In contrast to a regular $\mathrm{BH}$ concept admitting a horizon without a singularity [16-20], the conformally unimodular gauge changes the terminology completely. Namely, all its solutions have no horizon without requiring any exotic states of matter.

In principle, using the Dirac delta function and writing the density energy in equation (6) as $\rho(\boldsymbol{x})=e^{-3 \alpha} \delta^{(3)}(\boldsymbol{x})$ [40], one could consider the solutions (14), (15) as corresponding to the $\delta$-source, but such a consideration is rather formal because the equations of gravity are nonlinear, whereas the product of generalized functions cannot be defined correctly [43]. Some additional definition of the structure of the Dirac delta function is required to overcome this difficulty [44]. For instance, one could consider a physical model of delta-function in the form of a sphere of constant density, with the radius approaching zero along with the density tending to infinity. 

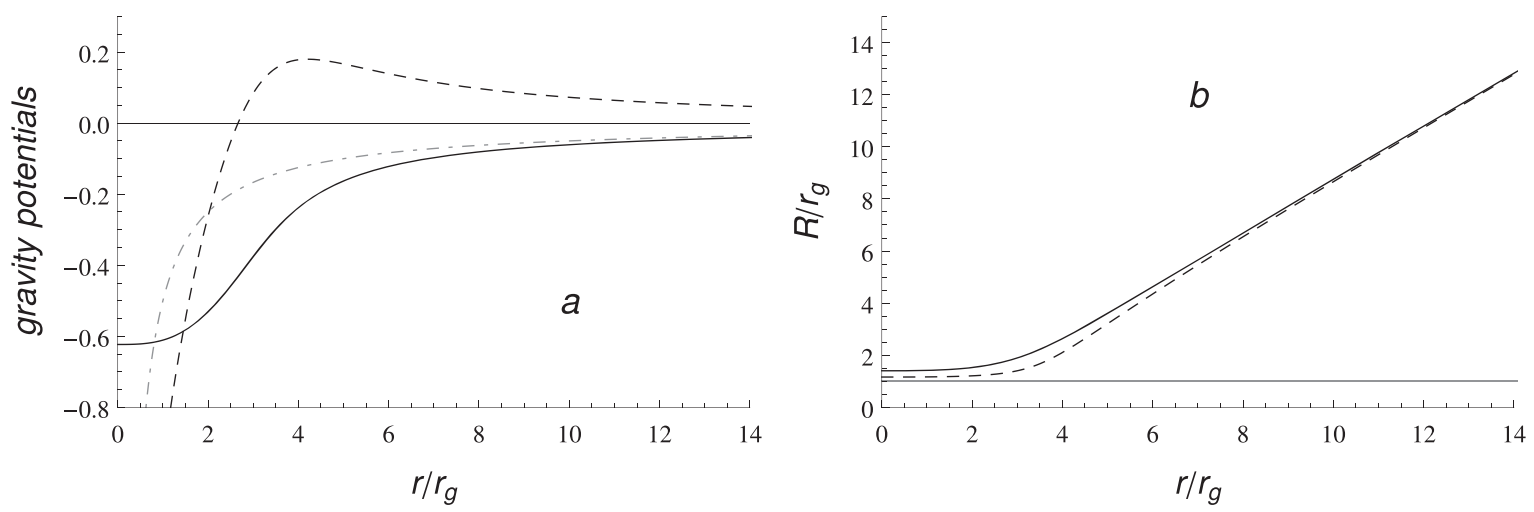

Figure 2. (a) 'Gravitational potentials' describing the metric (5). Solid and dashed lines correspond to $\alpha(r)$, and $\lambda(r)$, respectively. The dashed-doted line is the Newtonian potential $\varphi=-\frac{r_{g}}{2 r}$. (b) Coordinate transformation $R(r)$ mapping the metric (5), (23) to the canonical Schwarzschild form (22) for the different integration constant values $r_{0}$ in the expression (16). Solid and dashed lines correspond to $r_{0}=0$ and $r_{0}=3 r_{g}$, respectively. The level of $R=r_{g}$ is marked by the gray horizontal line.

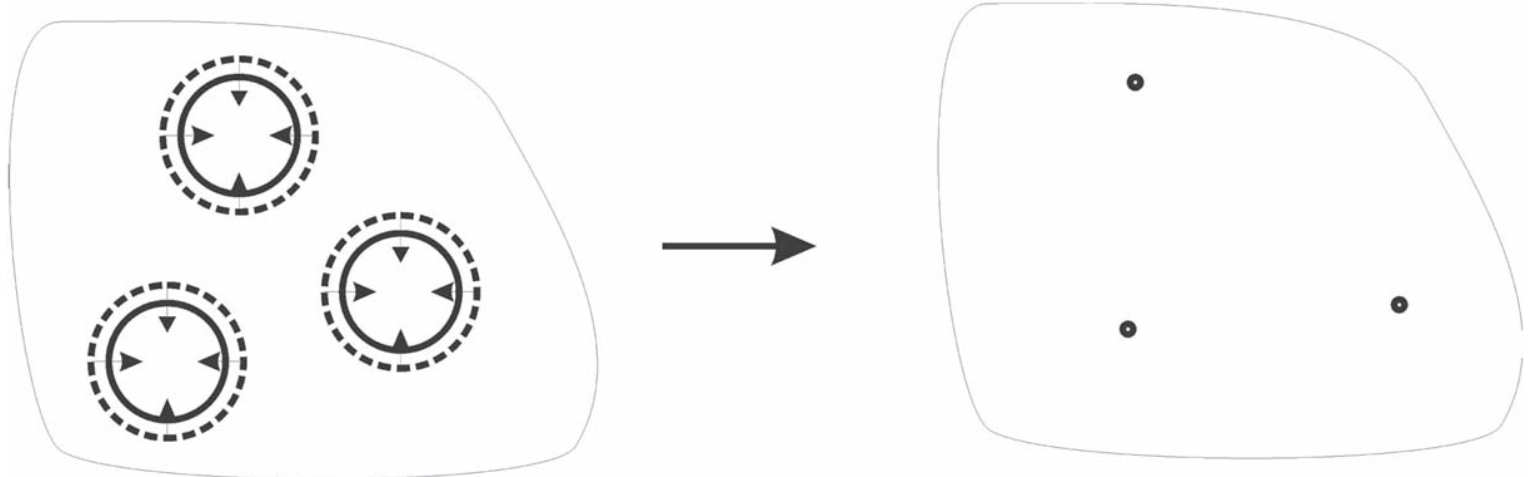

Figure 3. 'Squeezing' of the BHs of Schwarzschild horizons into the nodes with point masses.

\section{Compact objects of the constant density}

\subsection{Uniform compact object in the Schwarzschild metric}

The well-known Tolmen-Oppenheimer-Volkov equation (TOV) [5], which defines the maximal mass of a stable neutron star, written in the Schwarzschild type metric

$$
d s^{2}=B(R) d t^{2}-A(R) d R^{2}-R^{2} d \Omega,
$$

reads as:

$$
\begin{aligned}
p^{\prime}(R)= & -\frac{3}{4 \pi M_{p}^{2} R^{2}} \mathcal{M}(R) \rho(R)\left(\frac{1+4 \pi R^{3} p(R)}{\mathcal{M}(R)}\right) \\
& \times\left(1+\frac{p(R)}{\rho(R)}\right)\left(1-\frac{3 \mathcal{M}(R)}{2 \pi M_{p}^{2} R}\right)^{-1},
\end{aligned}
$$

where the function $\mathcal{M}(R)=4 \pi \int_{0}^{R} \rho\left(R^{\prime}\right) R^{\prime 2} d R^{\prime}$.

Although an ideal incompressible fluid seemed to be not existing in nature, an approximation of constant density [45] allows describing the general features of the compact physical objects. In this case $\mathcal{M}(R)=\frac{4 \pi}{3} \rho R^{3}$, the solution of equation (28) takes the form

$$
p(R)=\rho \frac{\sqrt{M_{p}^{2}-2 \rho R^{2}}-\sqrt{M_{p}^{2}-2 \rho R_{f}^{2}}}{3 \sqrt{M_{p}^{2}-2 \rho R_{f}^{2}}-\sqrt{M_{p}^{2}-2 \rho R^{2}}},
$$

where $R_{f}$ is the radius of an object. As it is seen from the formula (29), pressure turns to infinity at $R=\sqrt{4 M_{p}^{2} / \rho-9 R_{f}^{2}}$, that points to some limitations on the size of the object. A condition of pressure finiteness yields $4 M_{p}^{2} / \rho<9 R_{f}^{2}$, i.e., the size of an object has to be $R_{f}>\frac{9}{8} r_{g}$, where $r_{g}=\frac{3 m}{2 \pi M_{p}^{2}}, m=\mathcal{M}\left(R_{f}\right)=$ $\frac{4 \pi \rho R_{f}^{3}}{3}$.

\subsection{Shell compact object in the Schwarzschild metric}

Let us consider a more complex model of astrophysical object consisting of two immiscible and incompressible liquids with the densities $\rho_{1}$ and $\rho_{2}$. It is the simplest prototype for the neutron star with a non-uniform internal structure [46, 47].

Then, the function $\mathcal{M}(R)$ is written as

$$
\mathcal{M}(R)=\frac{4 \pi}{3}\left\{\begin{array}{cc}
\rho_{1} R^{3}, & R<R_{i}, \\
\rho_{2}\left(R^{3}-R_{i}^{3}\right)+\rho_{1} R_{i}^{3}, & R_{i}<R<R_{f}, \\
\rho_{2}\left(R_{f}^{3}-R_{i}^{3}\right)+\rho_{1} R_{i}^{3}, & R>R_{f} .
\end{array}\right.
$$

When $\rho_{1}$ is close to zero and $\rho_{2}=\rho$, the function (30) becomes 


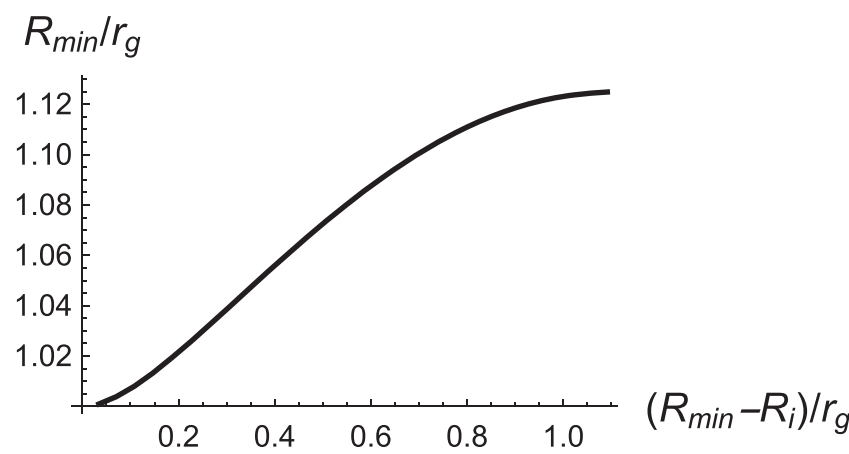

Figure 4. Minimum possible outer radius $R_{f}=R_{\min }$ in dependence on the thickness of a shell in the Schwarzschild metric (27).

$$
\mathcal{M}(R)=\frac{4 \pi \rho}{3}\left\{\begin{array}{cc}
0, & R<R_{i}, \\
R^{3}-R_{i}^{3}, & R_{i}<R<R_{f}, \\
R_{f}^{3}-R_{i}^{3}, & R>R_{f} .
\end{array}\right.
$$

The analytical solution of (28) for pressure with this $\mathcal{M}(R)$ is still cumbersome, however calculation shows softer condition for the pressure finiteness, which is shown in figure 4 . For a sufficiently thin shell $R_{f}$ approaches to $r_{g}$.

\subsection{Compact object in the conformally-unimodular metric}

4.3.1. Object of a star class. Modern observations of ultracompact BH-like objects, formed as a result of collapse of massive stars, give the maximum estimation of their masses of order of $m=15 \div 36 m_{\odot}[48,49]$. Let us consider the constant density objects in the metric (27) related by the coordinate transformation $R(r)=\exp (\alpha(r)-\lambda(r))$ with the metrics (14). A quantity $r_{f}$ denoting boundary of a matter in conformallyunimodular metric corresponds to $R_{f}=R\left(r_{f}\right)$ in metric of (23), while $R_{i}=R(0)$. Because the horizon is absent in this metric, nothing prevents $r_{f}$ to be smaller then $r_{g}$. The functions $\alpha(r), \lambda(r)$, $p(r)$ are defined by the equations (6)-(9) within a sphere occupied by matter. The boundary conditions at $r=r_{f}$ are given by linkage with the Schwarzschild solution (14), (16).

After solving of equations (6)-(9), the mass of an object can be recovered

$$
\begin{aligned}
m & =\frac{4 \pi}{3} \rho\left(R_{f}^{3}-R_{i}^{3}\right) \\
& =4 \pi \rho \int_{0}^{r_{f}} e^{3(\alpha(r)-\lambda(r))}\left(r \frac{d \alpha}{d r}-r \frac{d \lambda}{d r}+1\right) r^{2} d r
\end{aligned}
$$

which determines the Schwarzschild radius $r_{g}=\frac{3}{2 \pi} \frac{m}{M_{p}^{2}}$, appearing in the formulas (14) and (16).

Let us first discuss compact objects in the metric (5), (23) where the matter occupies a sphere with the size less or an order of the Schwarzschild radius (see figure 5), (a), (b). As could be expected, the potential $\alpha$, which was finite in the case of a point source, remains finite. The potential $\lambda$, which was infinite in the point where the point-like source was

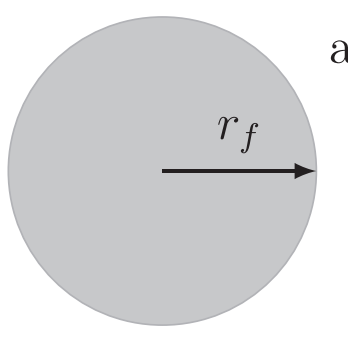

a

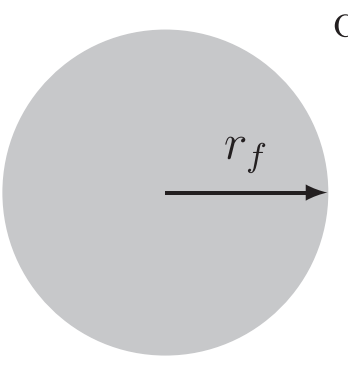

C

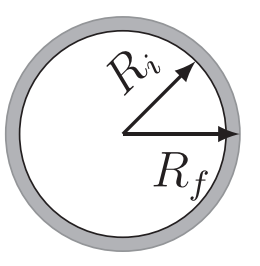

d

Figure 5. (a) A compact object of uncompressible fluid $\left(\rho_{0}=0.43 M_{p}^{2} r_{g}^{-2}\right)$ with the radius $r_{f}=2 r_{g}$ in the conformallyunimodular metric (23) looks as a shell (b) with the boundaries $R_{i}=R(0)=1.34 r_{g}$ and $R_{f}=R\left(r_{f}\right)=1.52 r_{g}$ in the Schwarzschild type metric (27). (c), (d) Low density object $\rho_{0}=5.0117 \times$ $10^{-10} M_{p}^{2} r_{g}^{-2}$ looks as a solid ball $R_{f} \approx r_{f}=1000 r_{g}$ in both metrics if parameter $r_{0}$ in (16) equals $r_{0}=-96.75$.

located, becomes finite inside a uniformly mass distribution within a ball.

The internal structure of a compact object in the metric (27) is defined by the internal $R_{i}$ and external $R_{f}$ radii, while there is only single external radius $r_{f}$ in the metric (23), and, besides, there is an additional parameter $r_{0}$ in the external Schwarzschild solution (14), (16). Thus, the meaning of this additional parameter $r_{0}$ becomes clear. Namely, it defines the internal structure of an object. It is not surprising that partial information about the pseudo-BH structure is contained in the Schwarzschild external solution in the form of parameter $r_{0}$ because no real $\mathrm{BH}$ in the conformally-unimodular metric exists.

Certainly, the pressure $P(R), R \in\left\{R_{i}, R_{f}\right\}$ obtained by the solution of the TOV equation matches the pressure recovered from equations (8), (9), (12) in the parametric form $p(r), R(r), r \in\left\{0, r_{f}\right\}$ as it is shown in figure 6.

4.3.2. Supermassive object. Recently, the existence of supermassive compact objects in galaxy nuclei was confirmed, and their masses were estimated as $m=$ $6.5 \times 10^{9} m_{\odot}$ [7]. Assuming the existence of some maximal density in nature $\rho_{\max } \simeq M_{p}^{4}$, after conversion to the units $M_{p}^{2} r_{g}^{-2}$, results in $\rho_{0}=\rho_{\max }=3.4 \times 10^{95} M_{p}^{2} r_{g}^{-2}$. For the conformally-unimodular metric, the size of this object turns out to be very small and, as calculations show, the potentials $\lambda$ and $\alpha$ inside a ball can be estimated by taking expressions (14), (16) for empty space (i.e., the boundary conditions affect $\alpha(r)$ stronger than the 'structure' of an object). Moreover, one 


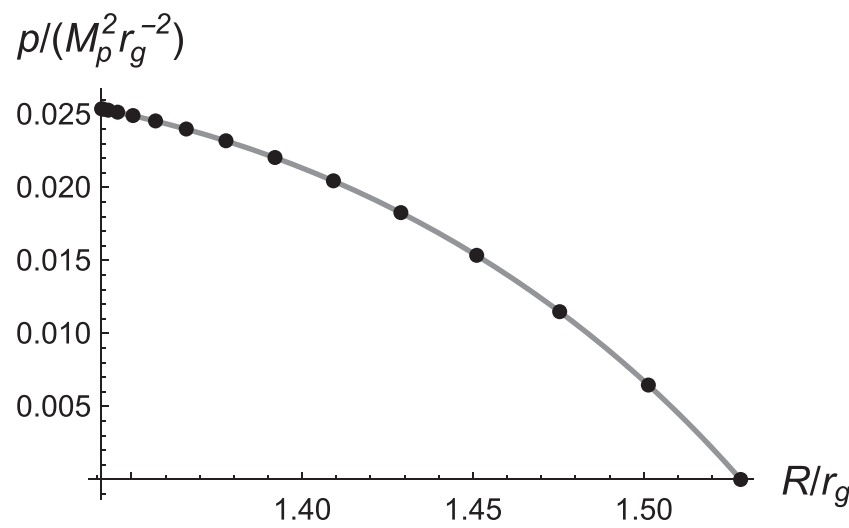

Figure 6. The pressure obtained by solving the TOV equation (points) and the equations (6), (7), (8), (9) (solid curve). The values of parameters correspond to figures $5(\mathrm{a}),(\mathrm{b})$.

has at a small $r / r_{g}$

$$
\alpha(r) \approx \ln \left(a_{0}\left(r_{0}\right)+\frac{r^{3}-r_{0}^{3}}{6 r_{g}^{3} k\left(r_{0}\right)}\right),
$$

because the value of $a$ tends to some constant $a_{0}$ at $r \rightarrow 0$. The parameters $a_{0}\left(r_{0}\right)$ and $k\left(r_{0}\right)$ are the functions of $r_{0}$. The expression (33) has been derived by the expansion of the function $f(a)$ into Taylor's series at the point $a_{0}$ up to the first order in $a-a_{0}$. After this expansion finding of the inverse function $f^{(-1)}$ becomes elementary. The value of $a_{0}$ is a root of the equation $f(a)-\frac{r_{0}^{3}}{6 r_{s}^{3}}=0$ and $k=f^{\prime}\left(a_{0}\right)$. Further, as an example, $r_{0}=0$ will be considered, when $a_{0} \approx 0.54$, and $k=25.2$.

The calculation of mass using (32) yields

$$
m \approx \frac{4 \pi}{3} \rho_{0} \frac{a_{0}}{k\left(1-a_{0}^{2}\right)^{4}} r_{f}^{3},
$$

giving the estimation for $r_{f} \approx 2.6 \times 10^{-32} r_{g}=1.5 \times$ $10^{16} M_{p}^{-1}$. The radius of a boundary surface in the Schwarzschild metric can be approximated from (24), (33)

$$
R(r) \approx \frac{r_{g}}{1-\left(a_{0}+\frac{r^{3}}{6 r_{g}^{3} k}\right)^{2}},
$$

which gives $R_{i}=R(0) \approx 1.4 r_{g}$.

As was already mentioned, it is possible to 'approach' closer to the Schwarzschild radius if to take another value of parameter $r_{0}$. The thickness of surface $\Delta R=R_{f}-R_{i} \approx$ $\left|\frac{d R(r)}{d r}\right|_{r \rightarrow r_{f}} \approx 7.5 \times 10^{-97} r_{g} \approx 4.3 \times 10^{-49} M_{p}^{-1}$. So small thickness $\Delta R$ of surface results from the hugeness of its area. The second equation of (12), using (33) and setting boundary condition $p\left(r_{f}\right)=0$ allow estimating the pressure

$$
p(r) \approx \frac{r_{f}^{3}-r^{3}}{r^{3}+6 a_{0} k r_{g}^{3}} \rho_{0} .
$$

The maximum of the pressure is $p \approx 0.07 M_{p}^{2} r_{g}^{-2}$, i.e., it is much lower than the density $\rho_{0}$, due to low potential gradient

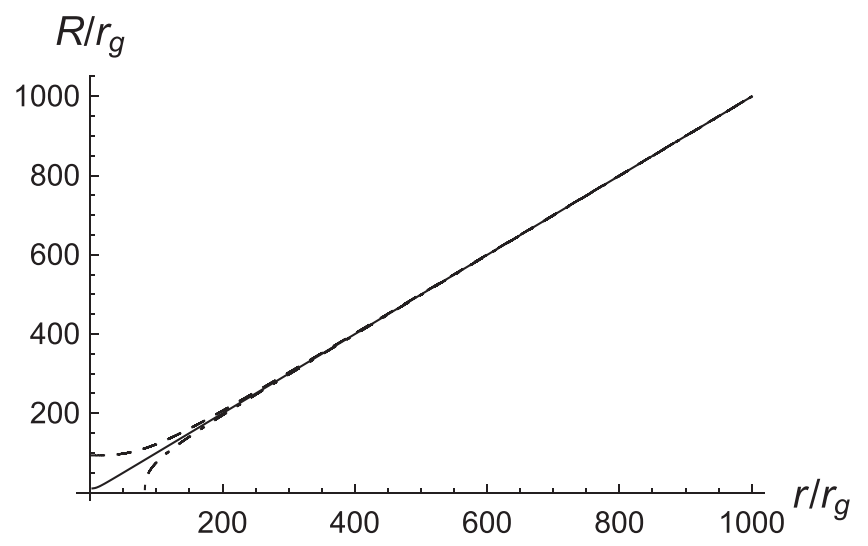

Figure 7. Dependence of $R(r)$ for a ball of $R_{f} \approx r_{f}=1000 r_{g}$ filled with the 'friable' matter at different values of the parameter $r_{0}$ in the external metric: $r_{0}=-120$ (dashed line), -96.75 (solid), -70 (dash-dotted). Density of matter is of $\rho_{0} \approx 5 \times 10^{-10} M_{p}^{2} r_{g}^{-2}$.

$\alpha(r)$ inside an 'eicheon' given by equation (33), or from the extremely small surface thickness $\Delta R$ in the terms of the TOV approach. This situation is analogous that in theory with massive graviton, where impenetrable surface exists before $r_{g}$ $[24,26]$ in the metric of (27) type. Since the real astrophysical objects must have the radii $>r_{g}$, this lifts the issue of the $\mathrm{BH}$ singularity in $[24,26]$.

\subsection{Low density objects}

Low density objects (recall, for example, that the Sun radius is $R_{f} \approx 236000 r_{g}$ ) illustrated in figures 5, (c), (d), which represents a solid ball in the Schwarzschild metric (27). Although they are not related to the compact objects but could be considered for the completeness of the picture. It turns out to be that, in this case, the value of parameter $r_{0}$ in external metric (16) is fixed by the requirement $r=0$ when $R=0$. As is shown in figure 7, the condition of $R=0$ at $r=0$ meets only if $r_{0}=-96.75$ for a non-compact object of the radius of $r_{f}=1000 r_{g}$. Then the value of $R(r)$ becomes almost the same with the $r$, as it is shown in figure 7. Thus, one may conclude that the 'friable' objects can also be described consistently in the conformally-unimodular metric.

In this context, it is interesting to imagine a low density object, but with an empty core surrounded by a firm 'artificial surface' composed from an incompressible liquid, for instance. Such an object can also be described in the conformally-unimodular metric, with the $r$-coordinate running from 0 to $r_{f}$ and $r_{0}$ should have a small negative value, that corresponds to an internal cavity in the Schwarzschild coordinates.

The difference between the 'friable' and compact dense objects is, that for the first one, internal cavity in the Schwarzschild metric could eliminate an by taking a larger value of $r_{0}$ in the metric (23). In contrast, for a dense object, the cavity in the Schwarzschild metric cannot be eliminated in any way. 


\section{Objects made of dust}

Let's consider the motion of a sample dust particle in the metric (23) in the neighborhood of $r=0$, where

$\alpha \approx$ const,$\quad \lambda=\alpha+\ln \left(\left(1-e^{2 \alpha}\right) r / r_{g}\right) \approx$ const $+\ln r$.

The radial geodesics satisfy the equation

$$
\ddot{\eta}=0, \quad \ddot{r}+2 \dot{r}^{2} / r=0,
$$

where a dot denotes a derivative over the proper time $s$. The solution of equation (38)

$$
r(\eta)=r_{i n}^{2 / 3}\left(r_{i n}-3 v\left(\eta-\eta_{i n}\right)\right)^{1 / 3}
$$

shows that the sample particle, placed initially at the point $r_{i n}$, $\eta=\eta_{\text {in }}$ and having the speed $v$ directed towards center, reaches the point $r=0$ for the finite time.

Qualitatively, the formation of objects with the equation of state of the dust type, i.e., having very low pressure, can be imagined as the radial falling of the dust particles in the 'eicheon' field. As a result, dust particles are accumulated in the vicinity of $r<r_{g}$, where the gradient of potential $\alpha$ is negligible, i.e., the gravitational field is absent. In this conformally-unimodular metric, 'eicheon' is similar to a trap because a particle needs to overcome the region of large potential to escape from such a trap. This picture is quite similar to those discussed in [10].

On the other hand, in the Schwarzschild type metric (27), a layer, where the dust particles are accumulated, is very thin. The thickness is determined by the residual pressure if to consider that some small pressure is still present. This picture resembles a a very thin surface discussed in [11, 25, 26, 50], where it originates from the non-zero mass of graviton.

\section{Conclusion}

We considered the conformally-unimodular gauge, which was chosen for the sake of avoiding the problem of vacuum energy. A requirement that the bulk vacuum energy $\rho_{v a c} \sim M_{p}^{4}$ does not influence the curvature of space-time leads to the gauge invariance violation and restricts the class of the possible metrics. That results in the absence of $\mathrm{BH}$ and the appearance of 'eiheons' instead. All the compact real astrophysical objects in this class of the metrics look like solid balls of different sizes without any singular surfaces ('horizons'). If such the compact objects $r_{f} \leqslant r_{g}$ are considered in the Schwarzschild metric, they look like a matter layer distributed over the impenetrable spherical shell with a radius greater than the Schwarzschild one.

We have considered only spherically symmetric solutions in the framework of the FVT, and see a further generalization of the presented model by taking into account the 'Eicheon' spinning and its 'non-hair' properties. Preliminary results concerning Kerr solution is sketched in the appendix.

\section{Appendix. The Kerr's solution into the conformally- unimodular metric}

Whereas the static and spherically symmetric 'eicheon' was above considered, the real astrophysical objects are spinning in nature. As it is well-known, the rotating $\mathrm{BH}$ has a region outside the Schwazshcild sphere known as the ergosphere [3], which plays a fundamental role in such phenomena as the Lense-Thirring (or frame-dragging) effect [51], the particle acceleration around a rotating black hole, and the Penrose process (i.e., the energy extraction from a rotating black hole) [52].

Therefore it is interesting to express the Kerr solution in the unimodular metric. The Kerr solution in the form of Boyer-Lindquist is written as $[3,53]$ :

$$
\begin{aligned}
d s^{2}= & \left(1-\frac{R r_{g}}{\wp^{2}}\right) d t^{2}-\frac{\wp^{2}}{R^{2}-r_{g} R+a^{2}} d R^{2}-\wp^{2} d \theta^{2} \\
& -\left(a^{2}+R^{2}+\frac{R r_{g} a^{2}}{\wp^{2}} \sin ^{2} \theta\right) \sin ^{2} \theta d \phi^{2} \\
& +\frac{2 r_{g} R a}{\wp^{2}} \sin ^{2} \theta d \phi d t,
\end{aligned}
$$

where $\wp^{2}=R^{2}+a^{2} \cos ^{2} \theta$.

To proceed with the unimodular metric, let's firstly to do the transformation to a new radial coordinate $r$ considering $R$ as a function $R(r, z)$, where, $z=r \cos \theta$. Writing $d R=$ $\partial_{r} R d r+\partial_{z} R(d r \cos \theta-r \sin \theta d \theta)$, one comes to

$$
\begin{aligned}
d s^{2}= & \left(1-\frac{R r_{g}}{\wp^{2}}\right) d t^{2}-\wp^{2} d \theta^{2} \\
& -\left(a^{2}+R^{2}+\frac{R r_{g} a^{2}}{\wp^{2}} \sin ^{2} \theta\right) \sin ^{2} \theta d \phi^{2} \\
& +\frac{2 r_{g} R a}{\wp^{2}} \sin ^{2} \theta d \phi d t \\
& -\frac{\wp^{2}\left(d r \partial_{r} R+\partial_{z} R(d r \cos \theta-d \theta r \sin \theta)\right)^{2}}{a^{2}+R^{2}-R r_{g}} .
\end{aligned}
$$

Then let us proceed with the isotopic coordinates $\boldsymbol{x}=\{x, y, z\}$

$$
\begin{aligned}
& x=\sqrt{a^{2}+r^{2}} \sin \theta \cos \phi, \\
& y=\sqrt{a^{2}+r^{2}} \sin \theta \sin \phi, \\
& z=r \cos \theta,
\end{aligned}
$$

by writing

$$
\begin{gathered}
\theta=\arccos \frac{z}{|x|}, \\
\phi=\arctan \frac{y}{x}, \\
r=\frac{1}{\sqrt{2}} \sqrt{x^{2}-a^{2}+\sqrt{4 a^{2} z^{2}+\left(x^{2}-a^{2}\right)^{2}}} .
\end{gathered}
$$

This leads to the following expression 
Table 1. Parity and time-reversal symmetry of the basic vectors.

\begin{tabular}{lccc}
\hline & $\boldsymbol{x}$ & $\boldsymbol{J}$ & $\boldsymbol{J} \times \boldsymbol{x}$ \\
\hline P- & - & + & - \\
T- & + & - & - \\
PT- & - & - & + \\
\hline
\end{tabular}

$$
\begin{aligned}
d s^{2}= & g_{t t} d t^{2}+g_{1}(d \boldsymbol{x} \boldsymbol{J})^{2}+g_{2}(d \boldsymbol{x} \boldsymbol{x})^{2} \\
& +g_{3}(d \boldsymbol{x} \boldsymbol{x})(d \boldsymbol{x} \boldsymbol{J})(\boldsymbol{J} \boldsymbol{x}) \\
& +g_{4}(\boldsymbol{J} \times \boldsymbol{x} \cdot d \boldsymbol{x})^{2}+g_{5} d t(d \boldsymbol{x} \cdot \boldsymbol{x} \times \boldsymbol{J}),
\end{aligned}
$$

Transformation properties of the basic vectors are given in a table 1 . The metric tensor considered (A.7) is parity and time reversal conserved quantity. In principle, P-, T- and PTsymmetries are violated in a nature, but here we restrict ourself only parity and time-reversal invariant case considering the transformation of only radial coordinate $R(r, z)$.

Conformally unimodular metric (1) requires that the coefficient under $d t^{2}$ in the third degree has to equal the determinant of the spatial metric (A.7). This gives the equation for the function $R(r, z)$ in the form of

$$
\partial_{r} R+\frac{z}{r} \partial_{z} R=\frac{\left(a^{2} z^{2}+r^{4}\right)\left(a^{2}+R^{2}-r_{g} R\right)^{1 / 2}\left(r^{2}\left(R-r_{g}\right) R+a^{2} z^{2}\right)^{3 / 2}}{\left(a^{2} z^{2}+R^{2} r^{2}\right)^{2}\left(a^{4} z^{2}+a^{2} R^{2}\left(r^{2}+z^{2}\right)+a^{2} r_{g} R\left(r^{2}-z^{2}\right)+R^{4} r^{2}\right)^{1 / 2}} .
$$

where $\boldsymbol{J}=\{0,0,1\}$ is an unit vector in the direction of the $\mathrm{BH}$ spin, $g_{i}$ are functions of $|\boldsymbol{x}|, z^{2}$, since $r$ is expressed through $|x|$ and $z$ through (A.5). As a result, one has:
From this point, the time variable becomes a conformal time $t=\eta$ of equation (1). To obtain solution of equation (A.8), first, it is convenient to consider the equation containing an

$$
\begin{aligned}
& g_{t t}=1-\frac{r^{2} r_{g} R}{r^{2} R^{2}+a^{2} z^{2}}, \\
& g_{1}=-\frac{\left(r^{2} R^{2}+a^{2} z^{2}\right)\left(\left(r^{2}-z^{2}\right)\left(\left(a^{2} z^{2}+r^{4}\right) \partial_{z} R+a^{2} r z \partial_{r} R\right)^{2}-r^{8} r_{g} R+r^{8} R^{2}+a^{2} r^{8}\right)}{\left(r^{2}-z^{2}\right)\left(a^{2} r z^{2}+r^{5}\right)^{2}\left(-r_{g} R+R^{2}+a^{2}\right)}, \\
& g_{2}=-\frac{r^{2}\left(r^{2} R^{2}+a^{2} z^{2}\right)\left(z^{2}\left(-r_{g} R+R^{2}+a^{2}\right)+r^{2}\left(r^{2}-z^{2}\right)\left(\partial_{r} R\right)^{2}\right)}{\left(r^{2}-z^{2}\right)\left(a^{2} z^{2}+r^{4}\right)^{2}\left(-r_{g} R+R^{2}+a^{2}\right)}, \\
& g_{3}=\frac{2 r\left(r^{2} R^{2}+a^{2} z^{2}\right)\left(\left(r^{2}-z^{2}\right) \partial_{r} R\left(\left(a^{2} z^{2}+r^{4}\right) \partial_{z} R+a^{2} r z \partial_{r} R\right)+r^{3}\left(r_{g} z R-z R^{2}-a^{2} z\right)\right)}{z\left(r^{2}-z^{2}\right)\left(a^{2} z^{2}+r^{4}\right)^{2}\left(r_{g} R-R^{2}-a^{2}\right)}, \\
& g_{4}=-\frac{r^{2}\left(a^{2}\left(r^{2}+z^{2}\right) R^{2}+a^{2} r_{g}\left(r^{2}-z^{2}\right) R+r^{2} R^{4}+a^{4} z^{2}\right)}{\left(a^{2}+r^{2}\right)^{2}\left(r^{2}-z^{2}\right)\left(r^{2} R^{2}+a^{2} z^{2}\right)}, \\
& g_{5}=-\frac{2 a r^{2} r_{g} R}{\left(a^{2}+r^{2}\right)\left(r^{2} R^{2}+a^{2} z^{2}\right)} .
\end{aligned}
$$

Vectors $\boldsymbol{N}$ and $\boldsymbol{P}$ appearing in (1) are $\boldsymbol{P}=0$ and $\gamma \boldsymbol{N}=-g_{5} \boldsymbol{x} \times \boldsymbol{J} / 2$. The FVT theory implies the restriction $\nabla(\nabla \cdot N)=0$ to vector $N$ because it is a Lagrange multiplier when the class of the metrics is restricted by (1). This restriction is evidently satisfied here by virtue of $\nabla \cdot N=0$.

The corresponding 3-metric tensor $\gamma_{\mu \nu}$ is

$$
\begin{aligned}
\boldsymbol{\gamma}= & g_{1} \boldsymbol{J} \otimes \boldsymbol{J}+g_{2} \boldsymbol{x} \otimes \boldsymbol{x}+\frac{g_{3}}{2}(\boldsymbol{x} \otimes \boldsymbol{J}+\boldsymbol{J} \otimes \boldsymbol{x})(\boldsymbol{J} \boldsymbol{x}) \\
& +g_{4}(\boldsymbol{J} \times \boldsymbol{x}) \otimes(\boldsymbol{J} \times \boldsymbol{x}),
\end{aligned}
$$

arbitrary function $G$

$$
\partial_{r} R+\frac{z}{r} \partial_{z} R=G(r, z)
$$

which has the formal solution

$$
R(r, z)=\int_{0}^{r} G\left(\xi, \xi \frac{z}{r}\right) d \xi+S\left(\frac{z}{r}\right)
$$

where $S(\cos \theta) \equiv \mathcal{R}(\theta)=\left.R(r, r \cos \theta)\right|_{r \rightarrow 0}$ is an arbitrary function determining the boundary condition at $r=0$. Thus, the final integral equation takes the form

$$
R(r, r \cos \theta)=\mathcal{R}(\theta)+\int_{0}^{r} \frac{\left(a^{2} \cos ^{2} \theta+\xi^{2}\right)\left(a^{2}+R^{2}-r_{g} R\right)^{1 / 2}\left(R\left(R-r_{g}\right)+a^{2} \cos ^{2} \theta\right)^{3 / 2}}{\left(a^{2} \cos ^{2} \theta+R^{2}\right)^{2}\left(a^{4} \cos ^{2} \theta+a^{2} R^{2}\left(1+\cos ^{2} \theta\right)+a^{2} r_{g} R \sin ^{2} \theta+R^{4}\right)^{1 / 2}} d \xi
$$




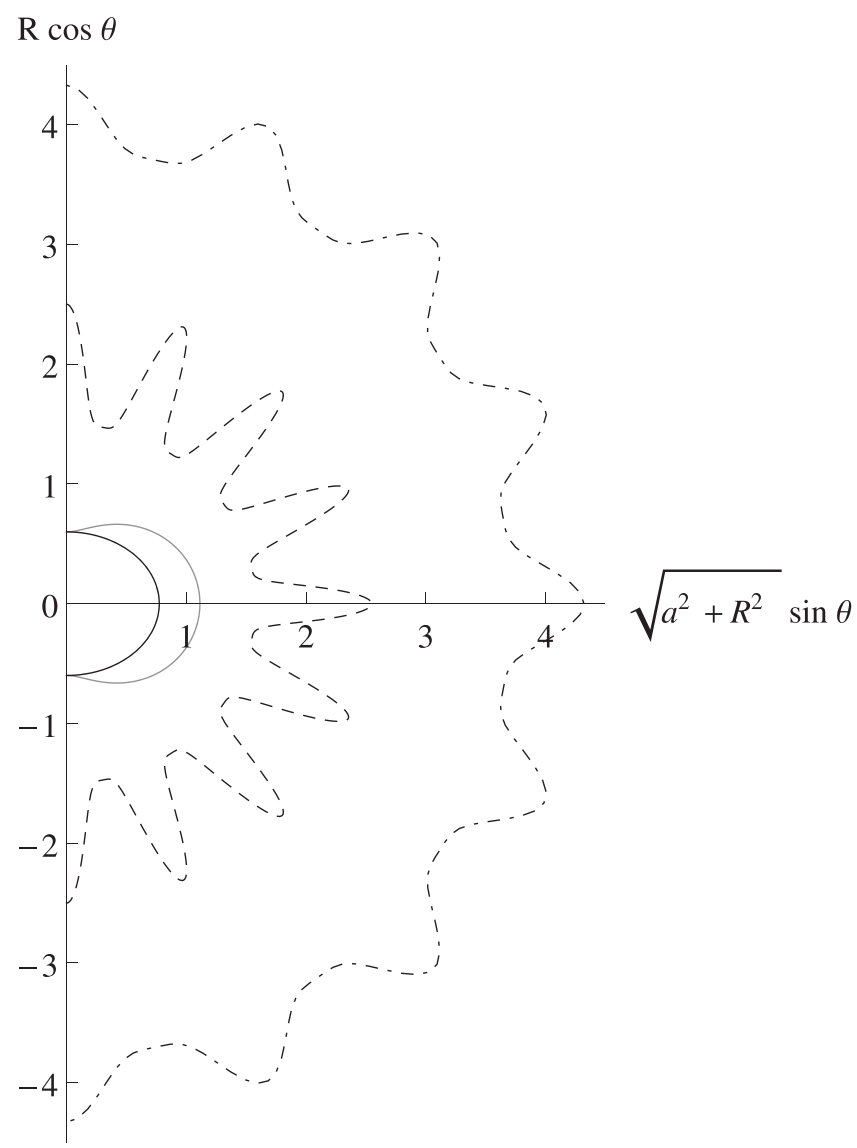

Figure A1. The form of the transformation coordinate surface $R(r, r \cos \theta)$ at $r=0$-dashed line, at $r=5$-dashed-dotted line. Horizon and ergosphere are shown by solid black and gray curves respectively. Units $r_{g}=1$ is used.

where it is implied that the function $R$ in the integrating expression has arguments $R(\xi, \xi \cos \theta)$. Equation (A.11) allows a numerical solution obtained iteratively in the form of the Neumann series. The zero-order approximation is $R=\mathcal{R}$ and implies that $R$ does not depend on $r$. Values in the fractional degrees in the integrating expression should be positive. This restricts $\mathcal{R}(\theta)$, namely, $a^{2}+\mathcal{R}^{2}-r_{g} \mathcal{R}>0$, and $\mathcal{R}\left(\mathcal{R}-r_{g}\right)+a^{2} \cos ^{2} \theta>0$, where last inequality corresponds to the ergosphere.

It means returning to a procedure discussed for the Schwarzschild solution when one chooses an arbitrary surface $\mathcal{R}(\theta)$ that surrounds the ergosphere and then shirks this surface into a point by the coordinate transformation $R(r, r \cos \theta)$ which satisfies the integral equation (A.11). In figure A1, the results of numerical solution are shown, where $\mathcal{R}(\theta)=r_{g}\left(3 / 2+\cos ^{4}(8 \theta)\right)$ and $a=0.49 r_{g}$ are taken as an example.

A point-like object is the idealization of the real compact astrophysical object. The real object is made of real matter with the interaction properties, including parity and timereversal symmetry. Although such a real entity can be formally described as a point, its physical properties preserve and contribute to the characteristics of such a point-like object. In this sense, the parity and time-reversal symmetry conservation/nonconservation are kinds of eicheon 'hairs'
[21]. Eicheon acquires 'hairs' because horizon disappears, which violates conditions of the Robinson theorem.

For $a>r_{g} / 2$, the singularity is naked even in the gauge (A.1), which could give rise to the concept of a 'BH electron' $[54,55]$. It seems that the conformally unimodular gauge is even more suitable for such interpretations, because, besides the naked singularity, there exists no ergosphere, and, possibly, no closed time-like geodesics. To obtain an 'elementary eicheon', one could choose the surface $\mathcal{R}(\theta)$ exactly along ergosphere.

\section{ORCID iDs}

S L Cherkas (10) https://orcid.org/0000-0002-6132-3052

\section{References}

[1] Weyl H 2018 Gravitation und elektrizität Das Relativitätsprinzip: Eine Sammlung von Originalarbeiten zur Relativitätstheorie Einsteins ed W Trageser (Berlin, Heidelberg: Springer Berlin Heidelberg) pp 239-52

[2] Novikov I D and Frolov V P 2001 Phys. Usp. 44291

[3] Chandrasekhar S 1983 The Mathematical Theory of Black Holes vol 1 (Oxford: Clarendon)

[4] Tolman R C 1939 Phys. Rev. 55364

[5] Oppenheimer J R and Volkoff G M 1939 Phys. Rev. 55374

[6] Abbott B P et al 2016 Phys. Rev. Lett. 116061102

[7] Akiyama K et al 2019 Astrophys. J. Lett. 8751

[8] Einstein A 1939 Ann. Math. 40922

[9] Popławski N J 2009 Phys. Lett. B 69073

[10] Chamseddine A H and Mukhanov V 2017 Eur. Phys. J. C 77183

[11] Logunov A A 1998 Relativistic Theory of Gravity (New York: Nova Science Publishers)

[12] Perez A 2017 Rep. Prog. Phys. 80126901

[13] Penrose R 1999 J. Astrophys. Astron. 20233

[14] Bardeen J M 1968 Non-singular general-relativistic gravitational collapse Proc. 5th Int. Conf. on gravitation and the theory of relativity ed V Fock et al (Tbilisi: Tbilisi University Press) p 174

[15] Bronnikov K A and Rubin S G 2013 Black Holes, Cosmology And Extra Dimensions (Singapore: World Scientific Publishing Co)

[16] Rodrigues M E and Silva M V D S 2018 J. Cosmol. Astropart. Phys. 2018 JCAP02(2018)025

[17] Roman T A and Bergmann P G 1983 Phys. Rev. D 281265

[18] Ayón-Beato E and Garcià A 1998 Phys. Rev. Lett. 805056

[19] Volkov M S and Galt'sov D V 1999 Phys. Rep. 3191

[20] Ayón-Beato E and Garcià A 2005 Gen. Rel. Grav. 37635

[21] Chruściel P T, Costa J L and Heusler M 2012 Living Rev. Relativity 157

[22] Kovács Z, Cheng K S and Harko T 2009 Mon. Not. Roy. Astron. Soc. 4001632

[23] Kiselev V, Logunov A and Mestvirishvili M 2010 Theor. Math. Phys. 164972

[24] Gershtein S, Logunov A and Mestvirishvili M 2009 Theor. Math. Phys. 1611573

[25] Vyblyi Y P 1991 Theor. Math. Phys. 88769

[26] Kalashnikov V L 2008 Centr. Eur. J. Phys. 680

[27] t‘Hooft G 1985 Nucl. Phys. B 256727

[28] Bousso R 2002 Rev. Mod. Phys. 74825

[29] Aste A and Trautmann D 2005 Can. J. Phys. 831001 
[30] Susskind L and Lindesay J 2005 An Introduction to Black Holes, Information and the String Theory Revolution: The Holographic Universe (New York: World Scientific)

[31] Blokhintsev D I 2009 Collected Papers vol 2 (Moscow: Fizmatlit)

[32] Weinberg S 1989 Rev. Mod. Phys. 611

[33] Blinnikov S I and Dolgov A D 2019 Phys. Usp. 62529

[34] Haridasu B S, Cherkas S L and Kalashnikov V L 2020 Fortschr. Phys. 682000047

[35] Wilczek F 1984 Phys. Rep. 104143

[36] Unruh W G 1989 Phys. Rev. D 401048

[37] Alvarez E 2005 J. High Energy Phys. JHEP03(2005)002

[38] Henneaux M and Teitelboim C T 1989 Phys. Lett. 222195

[39] Smolin L 2009 Phys. Rev. D 80084003

[40] Cherkas S L and Kalashnikov V L 2019 Proc. Natl. Acad. Sci. Belarus, Ser. Phys.-Math. 5583

[41] Arnowitt R, Deser S and Misner C W 2008 Gen. Relativ. Gravitation 401997

[42] Landau L D and Lifshitz E 1975 The Classical Theory of Fields vol 2 (Oxford: Butterworth-Heinemann)
[43] Vladimirov V S 1979 Generalized Functions in Mathematical Physics (Mir: Moscow)

[44] Katanaev M O 2013 Gen. Rel. Grav 451861

[45] Weinberg S 1972 Gravitation and Cosmology: Principles and Applications of the General Theory of Relativity (New York: Wiley)

[46] Potekhin A Y 2010 Phys. Usp. 531235

[47] Annala E, Gorda T, Kurkela A, Nättilä J and Vuorinen A 2020 Nat. Phys. (https://doi.org/10.1038/s41567-020-0914-9)

[48] Bulik T 2007 Nature 449799

[49] Miller M C and Yunes N 2019 Nature 568469

[50] Kalashnikov V L 2004 Perturbations of the spherically symmetric collapsar in the relativistic theory of gravitation: axial perturbations. I (arXiv:gr-qc/0405032)

[51] Lense J and Thirring H 1918 Zeit. Phys. 19156

[52] Bhat M, Dhurandhar S and Dadhich N 1985 J. Astrophys. Astron. 685

[53] Boyer R H and Lindquist R W 1967 J. Math. Phys. 8265

[54] Carter B 1968 Phys. Rev. 1741559

[55] Burinskii A 2008 Gravitation Cosmol. 14109 